\title{
NEW FORMULATION OF THE AXIOM OF CHOICE BY MAKING USE OF THE COMPREHENSION OPERATOR
}

\author{
KATUZI ONO
}

\section{Introduction}

We have introduced in our former work [1] and [2] the comprehension operator " $\{\cdot\}$ "1), which maps every binary relation to a binary relation. The definition of this operator

$$
x\left\{I^{\prime}\right) y \rightleftharpoons \forall s(s \in x \equiv s \Gamma y)^{2)}
$$

is remarkable in that it can be defined in any formal system having the membership relation $\in$, which is hereafter called the universal system and is denoted by $\mathbf{U}$. In this work, we would like to point out that the axiom of choice, in the strong sense as well as in the weak sense ${ }^{3)}$, can be formulated in an extremely simple style by making use of the comprehension operator.

One can make use of our formulations of the axiom of choice in most of the set-theoretical systems, i.e. theories of sets such as the systems of Zermelo [4] and of Fraenkel [5], theories of classes and sets such as the systems of v. Neumann [6], of Bernays [7]. and of Gödel [8], and also the theories of objects, OF and $\mathrm{OZ}$ of ourselves, introduced in [2] and [3], respectively.

To unify our notations in this work as far as possible, we would like to establish the following agreement:

We assume a binary relation $\in$ and a field of mathematical objects denoted

Received March 25, 1963.

1) The symbol " $\{\cdot)$ " is introduced in [1], and the name "comprehension operator" is introduced in [2]. In this work, we use mostly the same notations as those used in [1]. (Capital Greek letters as meta-symbols for binary relations in general. We use also notations of the forms $\Gamma \Delta, \bar{\Gamma}$, and $\Gamma \wedge \Delta$ for the relation product of $\Gamma$ and $\Delta$, for the inverse relation of $\Gamma$, and for the conjunction of $\Gamma$ and $\Delta$ (defined as $x(\Gamma \wedge \Delta) y=\cdot x \Gamma y \wedge$ $x \Delta y$ ), respectively. ( $\mathfrak{A} \rightleftharpoons \mathfrak{B}$ means here that $\mathfrak{A}$ is defined by $\mathfrak{B}$.)

2) In describing any axiom, any definition, or any theorem, we usually omit the universal quantifiers standing at its top and having the whole formula in its scope.

3) As for the exact meaning of these words, see Sections (2) and (3). 
by small Latin letters. We call every mathematical object denoted by these small Latin letters simply a set. The sets in this work can be regarded as sets in the theories of sets of Zermelo and of Fraenkel. They can be also regarded as sets in the theories of classes and sets of v. Neumann, of Bernays, and of Gödel. Namely, small Latin letters in this work can be regarded as set variables in these systems. One may use, in addition, capital Latin letters as class variables in these systems. Also, " $\in$ " can be regarded in $\mathbf{O Z}$ as its primitive notion proto-membership $\epsilon$, and, in the systems $\mathrm{OZ}$ and $\mathrm{OF}$, the small Latin letters can be regarded as their general object variables, originally denoted by capital Latin letters ${ }^{4)}$.

In this work, we introduce a special type of formulations of the axiom of choice, which can be described in the weakest system $\mathbf{U}^{5)}$ without any assumption. To examine mutual relations between our formulations and the popular forms of the axiom of choice, it seems better to examine them in stronger systems as occasion requires. Accordingly, we examine these relations in various settheoretical systems, naturally without assuming the axiom of choice. In this connection, we take up together with our systems $\mathbf{O F}$ and $\mathbf{O Z}$ the Fraenkel system (denoted by $\mathbf{S F}$ ) and the Gödel system (denoted by $\mathbf{C G}$ ), both without the axiom of choice, each as a representative of the respective kind of settheoretical systems.

In Section (1), we write down some definitions and theorems, which are valid in the universal system $\mathbf{U}$ together with some remarks. Preparing for Sections (2) and (4), Definitions and Theorems (1.1 D) - (1.11 R $)^{6)}$ have to do with mostly the notions of "left (or right) invariant" relations and of "left (or right) unique" relations. Definitions and Theorems (1.12 D) - (1.25 R) have to do with the usage of sets as binary relations, in preparation for Sections (3) and (5). Namely, we can define for any set $p$ two binary relations which are denoted by $\underline{p}$ and $\underset{\llcorner}{p}$ respectively. These relations $\underline{p}$ and $\underset{p}{p}$ coincide in SF and CG. They are called the binary relation defined by $p$. In CG, we can use also

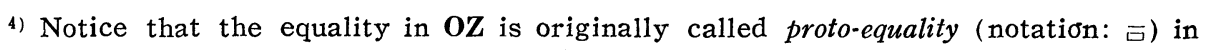
[3]. (Compare Definition (1.1 D) with [3]-(1.2.5) i.e. (1.2.5) of [3].)

5) Concerning formal systems, we say that a system is stronger than another system if and only if every theorem of the latter system is provable in the former system.

6) In numbering any definition or any remark, we attach the sign $\mathbf{D}$ or the sign $\mathbf{R}_{\mathbf{3}}$ respectively. 
any class $P$ as a binary relation, which is denoted by $\underline{P}$. (See Remark (1.25 R) and Section (4.), Formula (E).)

We show in Section (2), that the axiom of choice in the strong sense can be formulated as

$$
x \in y \rightarrow y \ni\{\eta) y
$$

where $\eta$ denotes a special binary relation which generates a universal choice function by the comprehension operator.

In Section (3), we show that the axiom of choice in the weak sense can be formulated as

$$
\exists r \forall x y(x \in y \in m \rightarrow y \ni\{\underset{r}{r}) y)
$$

or

(C)

$$
\exists r \forall x y(x \in y \in m \rightarrow y \ni\{\underline{r}) y) .
$$

We can introduce these axioms in any formal system stronger than $\mathbf{U}$. It is very remarkable that the axiom (C) implies the axiom of extensionality if we assume existence of the unit set for every set. (See (1.47 R) and (3.4).)

In Sections (4) and (5), we give a few generalizations and modulations of the axiom of choice. Namely, we give in Section (4) the axiom scheme

$$
\forall x y\left(x \Gamma y \rightarrow y \breve{\Gamma}\left\{\eta_{\Gamma}\right) y\right)
$$

as a generalization of the axiom of choice in the strong sense, where $\eta_{\Gamma}$ denotes a special binary relation depending on the binary relation $\Gamma$. This formula can be expressed more agreeably in $\mathbf{C G}$ as

$$
\forall P \exists Q \forall x y(x \underline{P} y \rightarrow y \underline{P}\{\underline{Q}) y) .
$$

In Section (5), we give a generalization and a modulation of the axiom (C). The one is namely the axiom scheme

$$
\exists r \forall x y(x \Gamma y \in m \rightarrow y \breve{\Gamma}\{\underline{r}) y) .
$$

This is a generalization of the axiom of choice anyway, but it seems that even the axiom of choice in the strong sense (A) does not imply this axiom scheme (F) even in very strong systems such as SF or CG. The other is the axiom

$$
\exists r \forall x y(x \underline{p} y \rightarrow \underline{y} \underline{\underline{p}}\{\underline{r}) y),
$$


which can be regarded as a modulation of (C). (See (5.6).)

In connection with our object theories, OF is practical except for that we have to define a rather complicated satellite relation before stating its axiom scheme. It is remarkable that we can replace this satellite relation by the simpler satellite relation of $\mathbf{O Z}$ when we assume the axiom $(\mathbf{C})$ or the axiom (G). We denote the systems having the replaced axiom scheme of $\mathbf{O F}$ and the axiom $(\mathbf{C})$ or the axiom $(G)$ by OMC or OMG respectively. (See (1.35 D), (1.36 D), (1.44 R), (5.4 D), and (5.7 R).) We can show that the system OMC as well as the system OMG is essentially nothing but the Fraenkel set-theory.

\section{(1) Preparatory formal theory developable without any assumption}

In this Section, we do not assume anything other than that our system has the notion "membership" (naturally denoted by " $\in$ "). All the definitions and theorems in this Section (except for Remark (1.25 R)) are valid in the universal system $\mathbf{U}$.

First of all, we define equality. However, in defining equality, two ways seem natural. The one is the way of defining equality by extensionality. The other is the way of defining equality by property. For convenience' sake, we call the former "equality" (See (1.1 D).) and the latter "identity" (See (1.2 D).)

(1.1 D) The relation $\{\in)$ is denoted by = and called equality.

(1.2D) $x=y \equiv \forall t(x \in t \equiv y \in t)^{\tau)}$. The relation $=$ is called identity.

(1.3 D) Any binary relation $\Gamma$ is called right (or left) invariant if and only if $\Gamma=\left(\right.$ or $=\Gamma$ ) implies $^{8)} \Gamma . \quad \Gamma$ is called invariant if and only if it is right invariant as well as left invariant.

(1.4 D) Any binary relation $\Gamma$ is called left (or right) unique if and only if $\Gamma \breve{\Gamma}$ (or $\breve{T} \Gamma$ ) implies =. $\Gamma$ is called unique if and only if it is right unique as well as left unique.

(1.5) Equality $=$ is reflexive, symmetric, and transitive. It is also invariant

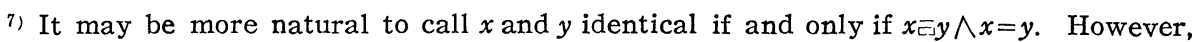
we adopt here simpler form, because $x_{\llcorner} y$ implies $x=y$ in the systems such as $\mathbf{O Z}$ or $\mathbf{O F}$. In SF (and also in CG for set variables $x$ and $y$ ) $x \overline{5} y$ and $x=y$ coincide.

8) We say that $\Gamma$ implies $\Delta$ if and only if $\forall x y(x \Gamma y \rightarrow x \Delta y)$ holds. 
and unique. (Proof. By (1.1 D), (1.3 D), and (1.4 D).)

(1.6) Identity $=$ is reflexive, symmetric, and transitive. (Proof. By (1.2 D).)

(1.7) Membership $\in$ is right invariant. (Proof. By (1.1 D) and (1.3 D).)

(1.8) If any binary relation $\Gamma$ implies another left (or right) unique binary relation, $\Gamma$ is also left (or right) unique. (Proof. By (1.4 D).)

(1.9) Any binary relation of the form $\{\Gamma)$ is left invariant as well as left unique. It is invariant if $\Gamma$ is right invariant. (Proof. By $(1.1 \mathrm{D}),(1.3 \mathrm{D})$, and (1. $4 \mathrm{D})$.)

(1.10) Any left unique and left invariant relation $\Gamma$ can be expressed in the form $\{\Delta)^{9}$. If $\Gamma$ is right invariant, we can take a right invariant $\Delta$ for this purpose. (As for the proof, see [2]-(2.31). Notice that this proof is valid in the universal system $\mathbf{U}$.)

(1.11 R) Remark. Sometimes, it looks advisable to take left (or right) uniqueness more strictly. Because we assume nothing corresponding to the axiom of extensionality, it is quite uncertain whether the condition $\forall x y u v(x \Gamma y$ $\wedge u \Gamma v \wedge y=v^{*} \rightarrow x=u$ ) holds for every left unique relation $\Gamma$. This is a stronger condition for a binary relation $\Gamma$, so we call any binary relation $\Gamma$ satisfying the above condition strongly left unique. Namely, any binary relation $\Gamma$ is called strongly left unique (or strongly right unique) if and only if $\Gamma=\bar{T}$ (or $\breve{\Gamma}=\Gamma$ ) implies equality. We can further define strong uniqueness in a natural way. We can prove by (1.4D) and (1.5) that any strongly left (or right) unique relation is left (or right) unique. We can further prove that any right (or left) invariant and left (or right) unique relation is strongly left (or right) unique. These two kinds of notions concerning uniqueness and strong uniqueness naturally coincide in systems such as SF or CG.

Next we define pairs and ordered pairs, and further we introduce binary relations defined by sets.

(1.12 D) $p\{x, y\} \rightleftharpoons \forall t(t \in p \equiv \cdot t=x \vee t=y)$. Any set $p$ satisfying $p\{x, y\}$ is called $a$ pair set of $x$ and $y$.

(1.13 D) $p\{x, y\} \equiv \forall t(t \in p \equiv \cdot t=x \vee t=y)$.

9) This means that we can express $\Delta$ in terms of $\Gamma$ so that $\forall x y(x \Gamma y \equiv x\{\Delta) y)$ holds. 
(1.14) $p\{x, y\} \wedge q\{u, v\} \cdot \rightarrow: p=q \equiv \cdot(x=u \wedge y=v) \vee(x=v \wedge y=u)$ and $\quad p\{x, y\} \wedge q\{u, v\} \cdot \rightarrow: p=q \equiv \cdot(x=u \wedge y=v) \vee(x=v \wedge y=u)$. (Proof. By (1.1 D), (1.5), (1.6), (1.12 D), and (1.13 D).)

(1.15 D) $p\langle x, y\rangle ₹ \exists u v(p\{u, v\} \wedge u\{x, x\} \wedge v\{x, y\})$. Any set $p$ satisfying $p\langle x, y\rangle$ is called an ordered pair of $x$ and $y$.

(1.16 D) $p\langle x, y\rangle \equiv \exists u v(p\{u, v\} \wedge u\{x, x\} \wedge v\{x, y\})$.

(1.17) $p\langle x, y\rangle \wedge q\langle u, v\rangle \cdot \rightarrow: p=q \equiv \cdot x=u \wedge y=v$. and $\quad p\langle x, y\rangle \wedge q\langle u, v\rangle \cdot \rightarrow: p=q \equiv \cdot x \equiv u \wedge y \equiv v$. (Proof. By (1.1D), (1.5), (1.6), (1.12 D), (1.13 D), (1.14), (1.15 D), and (1.16 D).)

(1.18D) $x \underline{r} y \equiv \exists p(p \in r \wedge p\langle x, y\rangle)$. We can regard $\underline{r}$ as a binary relation, which is called the binary relation defined by the set $r$ with respect to equality.

(1.19 D ) $x \underset{\square}{r} y \equiv \exists p(p \in r \wedge p\langle x, y\rangle)$. We can regard $\underset{\sim}{r}$ too a binary relation, which is called the binary relation defined by the set $r$ with respect to identity.

(1.20) $u\{x, y\} \vee u\{x, y\} \cdot \rightarrow \cdot x \in u \wedge y \in u$. (Proof. By (1.5), (1.6), (1.12 D), and (1.13 D).)

(1.21) $p\langle x, y\rangle \vee p\langle x, y\rangle \cdot \rightarrow \cdot x \in \in p \wedge y \in \in p$. (Proof. By (1.15 D), (1.16 D), and (1.20).)

(1.22) $x \underline{r} y \vee x r y \cdot \rightarrow \cdot x \in \in \in r \wedge y \in \in \in r . \quad$ (Proof. By (1.18 D), (1.19 D), and (1.21).)

(1.23) Any binary relation of the form $\underline{r}$ is invariant. Also, $=r=$ implies r. (Proof. By (1.3 D), (1.5), (1.6), (1.17), (1.18 D), and (1.19 D).)

(1.24) Any binary relation of the form $\{\underline{r})$ is invariant and left unique. Also, $\{\underline{r})=$ implies $\{\underline{r})$. (Proof. By (1.9) and (1.23).)

$(1.25 \mathrm{R})$ Remark. In CG, we can use any class $R$ as a binary relation, which is naturally defined by $x R y \equiv \exists p(p \in R \wedge p\langle x, y\rangle)$. Notice that $p\langle x, y\rangle$ and $p\langle x, y\rangle$ coincide in $\mathbf{C G}$.

Now we define generalized membership, inclusion, and generalized inclusion in preparation for introducing the satellite relations.

(1.26 D) $x \leqq y \equiv x=\in y . \quad$ (Generalized membership) 
(1.27 D) $x \subseteq y \rightleftharpoons \forall s(s \in x \rightarrow s \in y)$. (Inclusion, subset)

(1.28 D) $x \subseteq \bar{\subseteq} \equiv \forall s(s \hat{\in} x \rightarrow s \in \hat{E} y)$. (Generalized inclusion, generalized subset)

(1.29) $x \in y \rightarrow x \in \hat{E}$. (Membership implies generalized membership. Proof. By (1.5) and (1.26 D).)

(1.30) Generalized membership $\hat{\epsilon}$ is invariant. (Proof. By (1.5), (1.7), and $(1.26 \mathrm{D})$.)

(1.31) $x \subseteq y \rightarrow x \subseteq y$. (Inclusion implies generalized inclusion. Proof. By (1.5), (1.29 D), (1.27 D), and (1.28D).)

(1.32) Inclusion $\subseteq$ as well as generalized inclusion $\subseteq$ is reflexive, transitive, and invariant. (Proof. By (1.3 D), (1.27 D), and (1.28 D).)

(1.33) $x \in \subseteq y \rightarrow x \in y$ and $x \in \bar{\subseteq} y \rightarrow x \subseteq y$. (Proof. By (1.27 D) and (1.28D).)

(1.34) $x=y \equiv \cdot x \subseteq y \wedge y \subseteq x$. (Proof. By (1.1 D), and (1.27 D).)

Now we define the satellite relations of $\mathbf{O F}$ and of $\mathbf{O Z}$.

(1.35 D) $x \sigma y \equiv \forall p(\forall s(s \in \in p \equiv s \subseteq \in p) \wedge y \in \in p \cdot \rightarrow x \in \in p) . \quad$ (Satellite relation of $\mathrm{OF}$. Only $(1.26 \mathrm{D})$ and $(1.28 \mathrm{D})$ are necessary for stating this definition.)

(1.36 D) $x \sigma y \equiv \forall p(\forall s(s \in \in p \equiv s \subseteq \in p) \wedge y \in \in p \cdot \rightarrow x \in \in p)$. (Satellite relation of OZ. Only $(1.27 \mathrm{D})$ is necessary for stating this definition. As a fundamental notion, $\sigma$ can be defined in a simpler way than $\sigma$ ).

(1. 37) $x \in y \rightarrow x \sigma y, x \in y \rightarrow x_{\digamma \jmath} y, x \subseteq y \rightarrow x \sigma y, x \subseteq y \rightarrow x \sigma y, x\{=) y \rightarrow x \sigma y, x\{=) y$ $\rightarrow x \sigma y, x \sigma \sigma y \rightarrow x \sigma y$, and $x \sigma \sigma y \rightarrow x \sigma y$. (Broadly speaking, any member, any subset, and any unit set of a set are all satellites of the set, in the sense of OF as well as in the sense of $\mathrm{OZ}$. These satellite relations in both senses are also tranistive. Proof. By (1.1 D), (1.2 D), (1.26 D), (1.27 D), (1.28 D), (1.32), (1.33), (1.35 D), and (1.36 D).)

(1.38) $x=y \rightarrow x \sigma y$ and $x=y \rightarrow x \sigma y$. Also, $x=g=y \rightarrow x \sigma y$. (Proof. By (1.31), (1.34), (1.37), (1.2 D), (1.35 D), and (1.36 D).)

(1.39) $x \underline{r} y \vee x_{\square} r y=\rightarrow \cdot x_{\sigma} r \wedge y_{\sigma r} \wedge x_{\sigma} \sigma \wedge \wedge y_{\sigma} r . \quad$ (Proof. By (1.22) and (1.37).)

(1. 40) $(x\{\underline{r}) y \vee x(\underline{r}) y) \wedge(s\{\sigma) r \vee s\{g) r) \cdot \rightarrow x \subseteq s . \quad$ (Proof. By (1.27 D) and (1.39).) 
(1.41) $x\{\underline{r}) y \rightarrow x\{\hat{\epsilon}) x$. (Proof. By (1.23) and (1.26 D).)

Lastly, we give some remarks for later references.

(1.42 R) Remark. OF is the system having only one axiom scheme (OF)

$$
\exists p \cdot p\left\{\{\Gamma)_{\sigma}\right) m \text {. }
$$

(1.43 R) Remark. OZ is the system having only one axiom scheme $(\mathbf{O Z})$

$$
\exists p \cdot p\{\Gamma \wedge g) m \text {. }
$$

(1.44 R) Remark. Let us denote by OM (object theory with a mixed-type axiom-scheme) the system which have as its only one axiom scheme

$$
\exists p \cdot p\{(\Gamma) g) m \text {. }
$$

The system OM itself is not so attractive compared with $\mathrm{OF}$ or $\mathbf{O Z}$, because we have been unable to imbed any important set-theoretical system in it. However, the system turns out to be very remarkable when we assume the axiom (C) in it. (See Section (2).)

(1.45 R) Remark. According to (1.37), $g$ implies $\sigma$ in OF. As for the proof, see [2]-(5.8).

(1.46 R) Remark. $\forall m \exists p \cdot p\{=\Gamma \wedge g) m$ holds in $\mathrm{OF}$, in $\mathrm{OZ}$, and in OM. Namely, $\exists p \cdot p\left\{=r^{\prime} \wedge \sigma\right) m$ holds in OF as is shown in [2]-(4.2). Quite similarly, we can prove $\exists p \cdot p\{=\Gamma \wedge g) m$ in OM. Because $\exists p \cdot p\{=\Gamma \wedge \sigma) m$ implies $\exists p \cdot$ $p\{=\Gamma \wedge g) m$ in OF by (1.45 R), $\exists p \cdot p\{=\Gamma \wedge g) m$ holds in OF. The same formula holds also in $\mathbf{O Z}$, because it is a special case of the axiom scheme $(\mathbf{O Z})$.

$(1.47 \mathrm{R})$ Remark. In our terminology, the axiom of extensionality can be formulated as

$$
\forall x \cdot x\{(\hat{)} x .
$$

Namely, if we assume (EX), holds $\forall x y p(x=y \rightarrow \cdot x \in p \rightarrow y \in p)$ by (1.5) and (1.26 D). Consequently, $\forall x y(x=y \rightarrow \cdot \mathfrak{A}(x) \equiv \mathfrak{A}(y))$ is provable for any formula $\mathfrak{H}(\cdot)$ by making use of this fact and $(1.1 \mathrm{D})$. On the other hand, we can prove (EX) by (1.1D), (1.5), (1.26D), and (1.29), if we assume $\forall x y p(x=y \rightarrow \cdot$ $x \in p \rightarrow y \in p)$. $^{10)}$

10) Any $x$ satisfing $x\{\hat{E}) x$ is called normal in [2]. 


\section{(2) Strong form of the axiom of choice}

To make clear as far as possible what we have in mind as the axiom of choice in the strong sense, we state it at first in the ordinary terminology as follows :

$(2.1 \mathrm{~S})^{11)}$ We can assume that a function $\varphi^{12)}$, defined over the whole domain of sets other than the null set, satisfies $\forall x \cdot \varphi(x) \in x$.

Naturally, we can not expect to have such a universal choice-function $\varphi$ as a set. Such function may be regarded either as a class (in such theory of classes and sets as $\mathbf{C G}$ ) or as a special binary relation $\varphi$ introduced to define the function $\varphi$. In our terminology, the special binary relation $\varphi$ must be left unique, because $\varphi$ is assumed to be a function. So the axiom of choice (2.1S) can be characterized by the axioms $(2.2 \mathrm{~S})-(2.4 \mathrm{~S})$ :

(2.2 S) $x \varphi \breve{\varphi} y \rightarrow x=y \quad$ (The special binary relation $\varphi$ defines a function.).

(2.3 S) $x \in y \rightarrow \exists z \cdot z \varphi y$ (The domain of the function defined by the binary relation $\varphi$ includes the whole domain of sets other than the null set.)

(2.4 S) $x \varphi y \rightarrow x \in y$ (The value $x$ of the function defined by the binary relation $\varphi$ at any $y$ is a member of $y$.).

Combined with the axiom (2.2S), we can replace the axioms (2.3S) and (2.4 S) by the single weaker axiom

$(2.5 \mathrm{~S}) x \in y \rightarrow y \ni \varphi y$.

Namely, any binary relation $\Phi$ satisfying the conditions $(2.3 \mathrm{~S})$ and $(2.4 \mathrm{~S})$ satisfies the condition $(2.5 \mathrm{~S})$ on $\varphi$. On the other hand, for any binary relation $\Phi$ satisfying the condition $(2.5 \mathrm{~S})$ on $\varphi$, the binary relation $\Phi \wedge \in$ surely satisfies the conditions $(2.3 \mathrm{~S})$ and $(2.4 \mathrm{~S})$ on $\varphi$. Moreover, for any left unique $\emptyset$ i.e. for any $\Phi$ satisfying the condition (2.1S) on $\varphi$, the binary relation $\Phi \wedge \in$ can be also proved to be left unique i.e. to satisfy the condition $(2.2 S)$ on $\varphi$ by virtue of (1.4 D) and (1.8). Consequently, the axiom (2.1S) can be expressed by the two axioms $(2.2 \mathrm{~S})$ and $(2.5 \mathrm{~S})$.

\footnotetext{
11) In numbering a proposition merely stated, we attach the sign $\mathbf{S}$.

12) The function symbol $\varphi$ used in the form $\varphi(x)$ stands naturally outside our formal system. We use this kind of symbols olny for illustration.
} 
By making use of the comprehension operator, we can further unify the axioms $(2.2 \mathrm{~S})$ and $(2.5 \mathrm{~S})$ into the single extremely simple axiom

$$
x \in y \rightarrow y \ni\{\eta) y .
$$

Namely we can prove

(2.6) The axiom (A) is equivalent to the pair of axioms (2.2 S) and (2.5 S).

Proof. For any binary relation $\Phi$ satisfying the conditions $(2.2 \mathrm{~S})$ and $(2.5 \mathrm{~S})$, the binary relation $=\Phi$ is left unique and left invariant by $(1.3 \mathrm{D})$, (1.4 D), and (1.5). So, according to $(1.10),=\Phi$ can be expressed in the form \{1). It can be easily proved by (1.5), that the binary relation $\Lambda$ satisfies the condition (A) on $\eta$. Conversely, for any binary relation $\Phi$ satisfying the condition (A) on $\eta$, the binary relation $\{\Phi) \wedge \in$ can be proved to satisfy the condition (2.2 S) on $\varphi$ by virtue of (1.4D), (1.8), and (1.9), and it can be proved to satisfy also the condition $(2.5 \mathrm{~S})$ on $\varphi$ trivially.

Thus the axiom of choice in the strong sense can be stated in the form (A). Namely, if (A) holds, the binary relation $w(\{\eta) \wedge \in) z$ defines universal choice-function regarding it as a mapping $z$ to $w$.

\section{(3) Weak form of the axiom of choice}

In the terminology of the ordinary set-theory, we take the following statement as the axiom of choice in the weak sense:

(3.1 S) For any set $m$, there is a function $\varphi$ (called a choice function of $m$ ) satisfying $\forall x \cdot \varphi(x) \in x$ that is defined for all members of $m$ possibly with an exception of the null set.

In this case, the choice function $\varphi$ of $m$ could be represented by a set. However, it is not necessary to express the relation $y=\varphi(x)$ in the forms $y \underline{p} x$ or $y p x$. We might as well express the relation in the forms $y\{\underline{r}) x$ or $y\{\underline{r}) x$, as these forms have the advantage that binary relations of the forms $\{\underline{r})$ or $\{r)$ are automatically left unique. Adopting these forms, (3.1S) can be expressed in the forms

$$
\exists r \forall x y(x \in y \in m \rightarrow y \ni\{r) y)
$$

or

$$
\exists r \forall x y(x \in y \in m \rightarrow y \ni\{\underline{r}) y) .
$$


(3.2) The axiom (A) implies the axiom (B) if we assume $\forall m \exists p \cdot p\{\Gamma \wedge g) m^{13)}$ for every $\Gamma$.

Proof $/ \mathbf{A} \rightarrow \mathbf{b}^{14)}$.

A) Assume $\forall x y(x \in y \rightarrow y \ni\{\eta) y)$ and $\forall m \exists p \cdot p\{I \wedge g) m$, for every $\Gamma$.

b) ) $\forall m \exists r \forall x y(x \in y \in m \rightarrow y \ni\{\underline{r}) y) \quad / \mathbf{b A} \rightarrow$ be. bA) $\forall m$ !

bb) $\exists h k ! h(g) k\{g) m / \mathbf{A}$.

bC) Define $\Phi$ by $s \Phi t \equiv \exists u v(s\langle u, v\rangle \wedge u \eta v)$.

bd) $\exists r ! r\{\Phi \wedge \sigma) h \quad / \mathbf{A}$.

be)) $\forall x y(x \in y \in m \rightarrow y \ni\{\underline{r}) y) \quad$ /beA $\rightarrow$ bef. beA) $\forall x y ! x \in y \in m$.

beb) $\exists w ! y \ni w\{\eta) y \quad / \mathbf{b e A}, \mathbf{A}$.

bec)) $\forall s\left(s \in w \rightarrow s_{r} r y\right) \quad /$ bec $A \rightarrow$ becm. $\quad$ becA $) \quad \forall s ! s \in w$.

becb) $\quad s \eta y$ /becA, beb. becc) $\quad s \circlearrowleft m$ /becA, beb, beA, (1.37).

becd) $y$ gm /beA, (1.37).

bece) $\exists u v ! u\{s, s\} \wedge v\{s, y\}$ /A, becc, becd, (1.13D), (1.38).

becf) $u \subseteq k \wedge v \subseteq k$ /bece, becc, becd, bb, (1.13 D), (1.27 D), (1.38).

becg) $\exists z ! z\{u, v\} \quad / \mathbf{A}$, becf, (1.12 D), (1.37), (1.38).

bech) $z \subseteq h$ /becf, becg, bb, (1.12 D), (1.27 D), (1.37), (1.38).

beci） $z g h$ /bech, (1.37). becj) $z\langle s, y\rangle$ /bece, becg, (1.16 D).

beck) $z \emptyset h$ /becj, becb, bC. becl) $z \in r$ /beck, beci, bd.

becm) $\quad s r y$ /becl, becj, (1.19 D).

bed)） $\forall s(s$ r $y \rightarrow s \in w) \quad /$ bed $A \rightarrow \operatorname{bedg} ;$ beb. bedA) $\forall s ! s r y$.

bedb) $\exists z ! z \in r \wedge z\langle s, y\rangle / \operatorname{bedA},(1.19 \mathrm{D})$.

bedc) $\quad z \Phi h$ /bedb, bd. bedd) $\exists u v ! z\langle u, v\rangle \wedge u \eta v$ /bedc, bC.

bede) $u=s \wedge v=y \quad$ /bedb, bedd, (1.17), (1.5).

bedf) ) $\quad s \eta v \quad /$ bedf $A \rightarrow$ bedfg. bedfA) Define $\Delta$ by $f \Delta g \equiv f \eta v$.

bedfb) $\exists q ! q\{\Delta \wedge g) u / \mathbf{A}$.

bedfc) $u \Delta u \wedge u g u$ /bedf A, bedd, (1.5), (1.38).

bedfd) $u \in q$ /bedfb, bedfc. bedfe) $s \in q$ /bedfd, bede, (1.2 D).

bedff） $s \Delta u$ /bedfe, bedfb. bedfg) $s \eta v$ /bedff, bedfA.

13) This is not exactly the axiom scheme (OZ). For, by this assumption here, $\Gamma$ may be expressed in terms of the binary relation $\epsilon$ and $\eta$, whereas in $(\mathbf{O Z}), \Gamma$ should be expressed exclusively in terms of the primitive notion $\epsilon$.

14) Proofs of this type are described in PLK, the system of the classical predicate logic (such as Gentzen's LK, [9]) described in the way introduced in [1]. An example proof in PLK is given in [1], and the nickname PLK is introduced in [2]. 
bedg) sny /A, bedf, bede, (1.2 D), (1.5), (1.38); just as we deduced bedf from $A$, bedd, and bede.

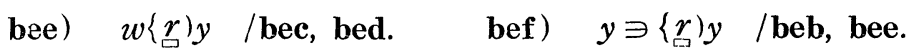

(3.3) The axiom (A) implies the axiom (C) if we assume the axiom scheme $\exists p \cdot p\{=I \wedge g) m^{15)}$ and $\forall x y(x=\eta=y \rightarrow x \eta y)$. As we can prove this theorem in a parallel way to the proof of the preceding theorem, we do not go into details of it.

We can hardly find out in $\mathbf{U}$ what difference lies between the axioms (B) and $(\mathbf{C})$. These axioms coincide in the usual set-theoretical systems such as SF, because these systems assume the axiom of extensionality. However, if we compare these axioms in object-theoretical systems such as $\mathbf{O Z}$ without assuming the axiom of extensionality, we can easily realize that the axiom $(\mathbf{C})$ is far the more powerful than the axiom (B). Namely,

(3.4) The axiom (C) implies the axiom of extensionality (EX) if we assume $\forall x \exists y \cdot y\{=) x$.

Proof $/ \mathbf{A} \rightarrow \mathbf{b}$ A) Assume the axiom (C) and $\forall x \exists y \cdot y\{=) x$.

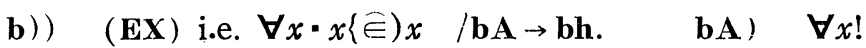

bb) $\exists m y ! m\{=) y\{=) x \quad / \mathbf{A}$. bc) $x \in y \in m \quad / \mathbf{b b},(1.5)$.

bd） $\exists r ! \forall s t(s \in t \in m \rightarrow t \ni\{\underline{r}) t) \quad$ /A. be $\quad \exists z ! y \ni z\{\underline{r}) y \quad /$ bc, bd.

bf) $z=x \quad /$ be, bb. $\quad$ bg $) \quad x\{\underline{r}) y \quad$ /be, bf, (1.5), (1.3 D), (1.9).

bh) $x\{\hat{E}) x /$ bg, (1.41).

(3.5 R) Remark. According to ( $\because 4)$, (1.37), (1.38), and (1.46 R), the equality notion coincides with the identity notion if we assume the axiom $(\mathbf{C})$ in any one of the systems $\mathbf{O F}, \mathbf{O Z}$, and $\mathbf{O M}$. We denote the system having one axiom (C) and one axiom scheme (OZ) by OZC, and the system having one axiom (C) and one axiom scheme (OM) by OMC. The satellite relations $\sigma$ and $\sigma$ coincide in these systems. One could naturally deal with also the system having one axiom (C) and one axiom scheme (OF). However, this system is proved to be equivalent to the system OMC. We prefer OMC to the

15) According to (1.46 R), it looks like as if (A) implies (C) in $\mathbf{O Z}$, in $\mathbf{O F}$, and also in $\mathbf{O M}$ as long as we assume that $\eta$ in $(\mathbf{A})$ is invariant. Exactly speaking, however, we can assert $\exists p \cdot p\{=\Gamma \wedge \sigma) m$ in these systems only when $\Gamma$ can be expressed in terms of $\in$ only. 
system, because the satellite relation of $\mathbf{O Z}$ can be introduced in a simpler way than the satellite relation of $\mathbf{O F}$.

(3.6 R) Remark. According to the theory developed in our work [3], one can easily realize that the Zermelo set-theory with the axiom of choice can be developed in ozc. In reality, the imbedding process can be much simplified than the imbedding process developed in [3]. For example, we can get rid of the weary steps in [3] for introducing the extensionality axiom. However, we do not go into details of this matter.

(3.7 R) Remark. The system OMC is equivalent to the Fraenkel set-theory with the axiom of choice but without the axiom of fundierung. We can prove this easily although we do not give here any detailed proof of it. We believe that the system of the axiom scheme (OM) and the axiom (C) is much simpler than the usual exposition of any axiom system of the Fraenkel set-theory, so we believe further that the system OMC is extremely practical as a starting point of the whole mathematical theories.

(3.8 R) Remark. The axiom (C) seems unprovable in the system OZB, i.e. the system having the axiom scheme $(\mathbf{O Z})$ and the axiom $(\mathbf{B})$ only. At present, it is an open question whether the system OZC can be proved to be consistent by assuming that the system OZB is consistent. ${ }^{16)}$

\section{(4) Modulation of the axiom of choice in the strong sense}

In our usual reasoning, inferences such as

(4.1S) If, for every natural number $n$, there exists an object $X_{n}$ bearing a given relation $\Gamma$ to $n$, there exists an infinite series of objects $X_{1}, X_{2}, \ldots$ satisfying the condition that, for every natural number $n, X_{n}$ bears the relation $\Gamma$ to $n$.

seem rather popular. Assuming the axiom of choice, this conclusion is surely true if for every natural number $n$ all the objects bearing the relation $\Gamma$ to $n$ form a set. However, we are apt to consider that this conclusion is valid even when we are not sure about whether, for every natural number $n$, all the objects bearing the relation $\Gamma$ to $n$ really form a set. This consideration

16) In the original manuscript, there had been an erroneous proof of the relative consistency of OZC, which was deleted in proof-reading. 
motivates us to formulate a general principle for inferences of this kind.

Corresponding to the axiom of choice in the strong sense, the principle can be taken in a very strong sense, which may be stated in the terminolgy of the ordinary set theory as follows:

(4.2 S) For any binary relation $\Gamma$, we can assume that a function $\varphi$, defined over the whole domain of sets other than the null set, satisfies $\forall x \cdot \varphi(x) I x$.

Also in this case, we can not expect to have such a choice funtion $\varphi$ as a set. Such function may be regarded either as a class in such theories of sets and classes as CG or as a special binary relation $\varphi_{\Gamma}$. In our terminology, $\varphi_{\Gamma}$ must be a left unique binary relation satisfying $\forall x y\left(x \Gamma y \rightarrow y \breve{\Gamma} \varphi_{\mathrm{r}} y\right)$. Just as in Section (2), we replace the left unique binary relation $\varphi_{\Gamma}$ by a relation of the form $\left\{\eta_{\Gamma}\right)$ which is surely left unique by (1.9). Accordingly, we formulate the above stated modulation (4.2S) of the axiom of choice in the strong sense as

$$
x \Gamma y \rightarrow y \breve{\Gamma}\left\{\eta_{\Gamma}\right) y .
$$

At the first glance, the axiom scheme (D) seems really simple, but it is not very agreeable because it contains the symbol $\eta_{\Gamma}$ for a special binary relation depending on the meta-symbol $\Gamma$ for a binary relation. It can be described more agreeably in $\mathbf{C G}$ as

$$
\exists Q \forall x y(x \underline{P} y \rightarrow y \underline{\underline{P}}\{\underline{Q}) y) .
$$

However, we can not set aside the form (D), because it leads to the interesting formula (F) as a modulation of the axiom of choice in the weak sense.

Anyway, the principles (D) or (E) seem really stronger than the axiom of choice even in the strong sense. The formula (A) is in fact a special case of the formula scheme (D). However, it seems impossible to prove (D) generally by assuming (A), even in such a strong system as SF. (Also, it seems impossible to prove $(\mathbf{E})$ by $(\mathbf{B})$ or $(\mathbf{C})$ in $\mathbf{C G}$.)

(5) Modulation of the axiom of choice in the weak sense

In modulating the axiom of choice in the weak sense, two ways seem possible. In the terminology of the ordinary set theory, we can describe them in the following two statements: 
(5.1 S) For any given set $m$ and for any binary relation $\Gamma$, there is a function $\varphi$ satisfying $\forall x \cdot \varphi(x) \Gamma x$ that is defined for all members $x$ of $m$ as far as there exists $y$ bearing the relation $y \Gamma x$.

(5.2 S) For any binary relation $\Gamma$ defined by a set, there is a function $\varphi$ satisfying $\forall x=\varphi(x) \Gamma x$ that is defined for all sets $x$ as far as there exists $y$ bearing the relation $y \Gamma x$.

Because, in ordinary set theories, the domain of any binary relation defined by a set is considered to be a set, we do not need to impose a condition in $(5.2 \mathrm{~S})$ that $x$ should be a member of a given set.

Just as in Section (3), the function $\varphi$ in (5.1S) or in (5.2S) might be as well expressed by a left unique binary relation of the forms $\{\underline{r})$ or $\{\underline{r})$.

\section{Along the line (5.1S):}

Just as we were in Section (3) led to the formulas (B) and (C) by the example of (A), we are led to the formula

$$
\exists r \forall x y(x \Gamma y \in m \rightarrow y \breve{\Gamma}\{\underline{r}) y)
$$

by the example of (D) as a matter of course. This formula is really remarkable not only because it can be regarded as a generalization of the formula (C) but because of its extremely extensive character. We could naturally introduce the formula $\exists r \forall x y(x \Gamma y \in m \rightarrow y \breve{\Gamma}\{r) y)$ as a modulation of the formula (B). However, this formula does not seem so serviceable as the formula (F).

The formula (F) naturally does not imply the formula (A). On the other hand, even in such a strong system as SF, it seems impossible to prove (F) by (A). Accordingly, the formula (F) can be regarded as another genaralization of the axiom of choice in the weak sense.

(5.3) The axiom scheme (F) implies the axiom (C).

(5.4D) The system having the pair of axiom schemes (OZ) and (F) is denoted by OZF.

(5.5) The system OZF is stronger than OMC; so, according to (3.7 R), it is stronger than the Fraenkel set-theory without the fundierung axiom. To prove this theorem, we have only to prove the axiom scheme (OM) in OZF, because we can prove $(\mathbf{C})$ in $\mathbf{O Z F}$ by virtue of $(5,3)$. 
Proof of $(\mathrm{OM})$ in $\mathbf{O Z F} / \mathbf{A}, \mathbf{B}, \mathbf{C} \rightarrow \mathbf{k}$. A) Assume $(\mathrm{OZ})$ and $(\mathbf{F})$.
B) $\quad \Gamma$ be any binary relation.
C) $\forall m$ !
d) $\exists h ! h(g) m \quad / \mathbf{A}$.
e) $\exists r ! \forall x y(x\{\Gamma) y \in h \rightarrow y(\Gamma\}\{\underline{r}) y)$
/A. f) $\exists q ! q\{g) r / \mathbf{A}$.
g)) $\forall x\left(x\{\Gamma) g m \rightarrow x_{\sqsubset \square} q\right) \quad / \mathrm{gA} \rightarrow \mathrm{gh}$.
gA) $\forall x ! x\{\Gamma)_{g} m$.
gb) $\exists y ! x\{\Gamma) y g m \quad / g \mathbf{A}$.
gc) $x\{\Gamma) y \in h \quad / g b, d$.
gd) $\exists z ! y(\Gamma\} z\{\underline{r}) y \quad$ /ge, e. $\quad$ ge $) \quad x=z \quad$ /gc, gd, (1.9).
gf) $x\{\underline{r}) y \quad / g e, g d,(1.9) . \quad$ gg) $x \subseteq q$ /f, gf, (1.40).
gh) $\quad x g q / \mathrm{gg},(1.37)$.
H) Define $\Delta$ by $x \Delta y \equiv x\{\Gamma) \sigma m . \quad$ i) $\exists p ! p\{\Delta \wedge g) q \quad / \mathbf{A}$.
j) $\quad p\{\Delta) m / \mathbf{g}, \mathbf{H}, \mathbf{i} . \quad$ k) $p\left\{\{\Gamma)_{g}\right) m / \mathbf{j}, \mathbf{H}$.

Along the line (5.2S):

As the second modulation of the axiom of choice in the weak sense, we have the proposition

$$
\exists r \forall x y(x \underline{p} y \rightarrow y \underline{\underline{p}}\{\underline{r}) y),
$$

which can be stated in any system having the membership relation. We could naturally introduce another modulation $\exists r \forall x y(x p y \rightarrow y \breve{p}\{\underset{r}{r}) y)$ which can be also stated in any system having the membership relation. However, we do not discuss on this formula because it does not seem so serviceable as $(\mathbf{G})$.

(5.6) The axioms (C) and (G) are mutually equivalent as long as $\forall m \exists p \cdot$ $p\{=\Gamma \wedge g) m$ holds for every $\Gamma$.

Proof $/ \mathbf{A} \rightarrow \mathbf{b}$, c. $\quad$ A ) Assume $\forall m \exists p \cdot p\{=\Gamma \wedge g) m$ for every $\Gamma$.

b) ) (C) implies (G) /bA $\rightarrow$ bd. bA) Assume (C).

bb) $\forall x \exists y \cdot y\{=) x / \mathbf{A},(1.5),(1.38)$.

bc) According to (3.4), the extensionality axiom (EX) holds by $\mathbf{A}$ and bb. Consequently, we have no need to draw any distinction between $\epsilon$ and $\hat{\epsilon}$, or between $\sigma$ and $\sigma$. Also, by virtue of $(1.47 \mathrm{R}), \mathbf{A}$ implies $\forall m \exists p \cdot p\{I \wedge \sigma) m$ for every $\Gamma$.

bd)）（G） / bdA $\rightarrow$ bdf. bdA $\quad \forall p$ !

bdb) $\exists m s q ! m\{\sigma) s\{\sigma) q\{\sigma) p \quad /$ bc.

bdc) $\exists u ! \forall x z(x \in z \in s \rightarrow z \ni\{\underline{u}) z) \quad /$ bA.

bdD) Define $\Gamma$ by $k \Gamma a \rightleftharpoons \exists x y(k\langle x, y\rangle \wedge x u\langle p) y)$. 
bde) $\exists r ! r\left(I^{\prime} \wedge \sigma\right) m \quad /$ bc.

bdf)) $\forall x y(x \underline{p} y \rightarrow y \underline{p}\{\underline{r}) y) \quad /$ bdfA $\rightarrow$ bdfl. bdfA） $\forall x y$ ! $x \underline{p} y$.

bdfb) $\exists z ! \forall t(t \in z \equiv \cdot t \underline{y} y \wedge t \sigma p) \quad /$ bc. $\quad$ bdfc) $\quad z\{\underline{p}) y \quad /$ bdfb, (1.39).

bdfd) $\quad z \subseteq q$ /bdfc, bdb, (1.4.0). bdfe) $z \in s$ /bdfd, bdb, (1.37).

bdff) $\quad x \in z \quad$ /bdfA, bdfc. bdfg) $\exists w ! z \ni w\{\underline{u}) z$ /bdff, bdfe, bde.

bdfh ) $y \underline{p} w$ /bdfc, bdfg.

bdfi)) $\forall t(t \in w \rightarrow t \underline{r} y) \quad$ /bdfiA $\rightarrow$ bdfin. bdfiA) $\forall t ! t \in w$.

bdfib) $t \underline{u z}$ /bdfiA, bdfg. bdfic) $t \underline{u}\{\underline{p}) y$ /bdfib, bdfc.

bdfid） $\quad z \sigma q$ /bdfe, bdb. bdfie) $t \sigma q$ /bdfiA, bdfg, bdfid, (1.37).

bdfif) $y_{\sigma q} /$ bdf A, bdb, (1.37), (1.39).

bdfig) $\exists f g ! f\{t, t\} \wedge g\{t, y\}$ /bdfie, bdfif, bc, (1.12 D), (1.38).

bdfih) $f_{\sigma s} \wedge \mathbf{g}_{\sigma} s \quad$ /bdfig, bdfie, bdfif, bdb, (1.27 D), (1.37), (1.38).

bdfii) $\exists k ! k\{f, \mathrm{~g}\} \quad$ /bdfih, bc, (1.12 D), (1.38).

bdfij) $k\langle t, y\rangle$ /bdfii, bdfig, (1.15 D).

bdfik) $\quad k T m$ /bdfij, bdfic, bdD.

bdfil) $k \sigma m$ /bdfii, bdfih, bdb, (1.27 D), (1.37), (1.38).

bdfim) $\quad k \in r$ /bdfik, bdfil, bde. bdfin) tray /bdfim, bdfij, (1.18 D).

bdf j) ) $\forall t(t \underline{r} y \rightarrow t \in w) \quad(\mathbf{b d f j A} \rightarrow$ bdfji. $\quad$ bdfjA $) \quad \forall t ! t \underline{r} y$.

bdfjb) $\exists k ! k \in r \wedge k\langle t, y\rangle /$ bdf jA A, (1.18 D).

bdfjc) $k \Gamma m /$ bdfjb, bde.

bdfjd) $\exists f g ! k\langle f, g\rangle \wedge f \underline{u}\{\underline{p}) g \quad /$ bdfjc, bdD.

bdfje ) $f=t \wedge g=y \quad /$ bdfjd, bdfjb, (1.5), (1.17).

bdfjf) $\exists v ! t \underline{u} v\{\underline{p}) y \quad /$ bdfje, bdfjd, (1.3 D), (1.5), (1.23), (1.24).

bdfjg) $\quad v=z$ /bdfjf, bdfc, (1.4 D), (1.24).

bdfjh) $\quad t u z$ /bdfjf, bdfjg, (1.3 D), (1.23). bdfji) $t \in w \quad /$ bdfjh, bdfg. bdfk) $\quad w\{\underline{r}) y \quad /$ bdfi, bdfj. $\quad$ bdfl) $\quad y \underline{p}\{\underline{r}) y \quad /$ bdfh, bdfk.

c) ) (G) implies (C) /cA $\rightarrow$ cb. $\quad$ cA) Assume (G).

cb) ) (C) $/$ cbA $\rightarrow$ cbi. cbA $) \quad \forall m$ !

cbB) Define $\Gamma$ by $f \Gamma g ₹ \exists x y(f\langle x, y\rangle \wedge x \in y \in m)$.

cbc) $\exists q n ! q\{g) n\{g) m \quad / \mathbf{A},(1.37),(1.38) . \quad$ cbd) $\quad \exists p ! p\{=\Gamma \wedge g) q \quad / \mathbf{A}$.

cbe) $\quad \Gamma$ is left invariant /cbB, (1.3 D), (1.17).

cbf)) $\quad \forall f(f I q \rightarrow f g q) \quad / \mathbf{c b f} \mathbf{A} \rightarrow \mathbf{c b f g} . \quad \operatorname{cbf} \mathbf{A}) \quad \forall f ! f \Gamma q$.

cbfb) $\exists x y ! f\langle x, y\rangle \wedge x \in y \in m \quad / \mathbf{c b f} \mathbf{A}, \mathbf{c b B}$.

cbfe) $\quad x_{\sigma} m \wedge y_{\sqsubset} m \quad / \mathbf{c b f b},(1.37)$. 


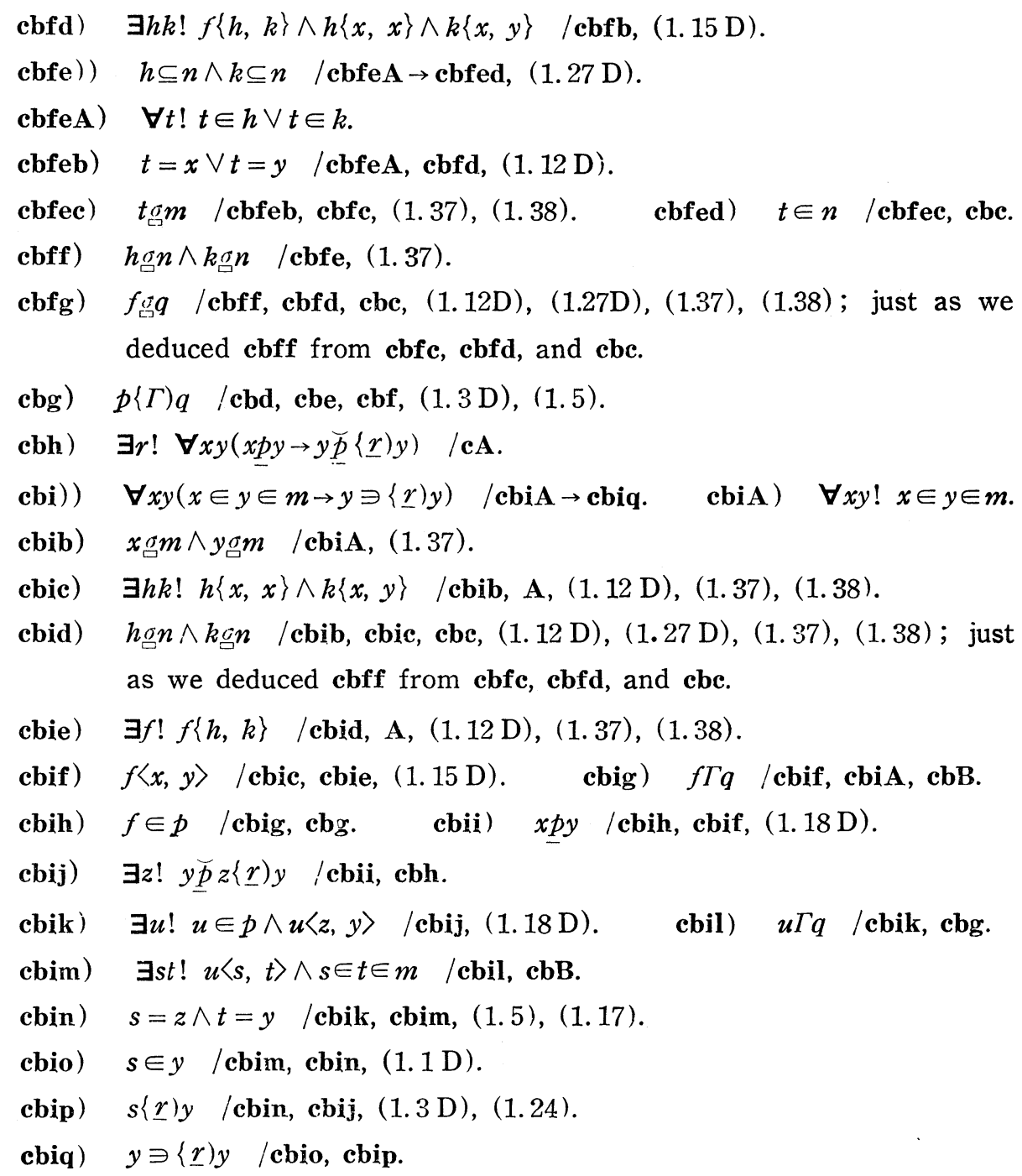

(5.7 R) Remark. According to (1.46R) and (5.6), we know that the systems OZC and OZG as well as the systems OMC and OMG are mutually equivalent. Here we denote by OZG and OMG the systems which have the axiom $(\mathbf{G})$ together with the axiom scheme $(\mathbf{O Z})$ or the axiom scheme (OM), respectively.

\section{REFERENCES}

[1] K. Ono, On a practical way of describing formal deductions. Nagoya Math. Journal. vol, 21, pp. 115-121 (1962), 
[2] K. Ono, A stronger system of object theory as a prototype of set theory. Nagoya Math. Journal, vol. 22, pp. 119-167 (1963).

[3] K. Ono, A theory of mathematical objects as a prototype of set theory. Nagoya Math. Journal, vol. 20, pp. 105-168 (1962).

[4] E. Zermelo, Untersuchungen über die Grundlagen de Mengenlehre, I, Math. Ann., vol. 65, pp. 261-281 (1908).

[5] A. Fraenkel, Einleitung in die Mengenlehre, 1928 (3. Aufl.)

[6] J. v. Neumann, Die Axiomatisierung der Mengenlehre, I, J. für reine u. angew. Math., vol. 154, pp. 219-240 (1925). Die Axiomatisierung der Mengenlehre. Math. Z., vol. 26, pp. 1-46 (1927).

[7] P. Bernays, A system of axiomatic set theory, Part I-VII; Symbolic Logic; I, vol. 2, pp. 65-77 (1937); II, vol. 6, pp. 1-17 (1941), III, vol. 7, pp. 65-89 (1942); IV, vol. 7, pp. 133-145 (1942); V, vol. 8, pp. 89-106 (1943), VI, vol. 13, pp. 65-79 (1948); VII, vol. 19, pp, 81-96 (1954).

[8] K. Gödel, The consistency of the axiom of choice and of the generalized continuum hypothesis with the axioms of set theory. Annals of Mathematics Studies, No. 3, 1940.

[9] G. Gentzen, Untersuchungen über das logischen Schliessen. Math. Z., vol. 39, pp. 176-210, 405-431 (1935). 